\title{
una teoria de la flexión con esfuerzo cortante en piezas de hormigón armado
}

J. A. LOPEZ JAMAR Y J. J. TIRADO CRUZ, ingenieros de caminos

En la Monografía número 221, el Instituto Eduardo Torroja de la Construcción y del Cemento ha publicado recientemente el trabajo arriba titulado, original de los ingenieros citados. Greemos constituye un interesante intento de explicar el comportamiento del hormigón armado en el caso de existir esfuerzos cortantes, simultáneamente con la flexión simple o compuesta. Actualmente se están realizando una serie de ensayos de rotura de vigas, en nuestros Laboratorios y bajo el patronato del Instituto, para contrastar esta teoría. Pero como consideramos interesará el asunto a muchos de nuestros lectores, exponemos a continuación un resumen de los
principales puntos de aquélla y su comparación con los criterios clásicos que se han venido siguiendo hasta ahora.
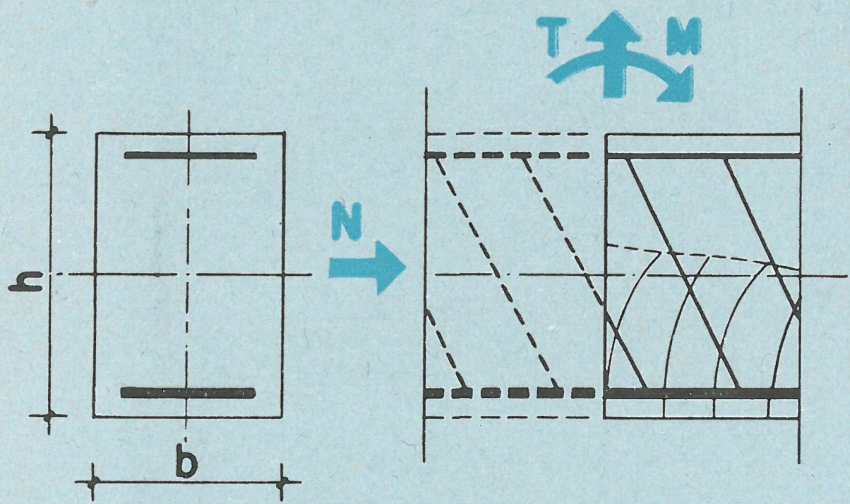

Ante la falta de precisión teórica y las evidentes contradiciones que se encuentran en los criterios clásicos y en algunas teorias recientemente aparecidas, y ante la falta de unidad de criterios que se observa en las reuniones internacionales que se ocupan de este probiema, si se examinan con detenimiento las diversas teorías se encuentra casi con carácter general que son incompletas por alguna de las siguientes causas:

No considerar todas las clases de deformaciones que aparecen en la pieza.

No cumplirse la compatibilidad de las deformaciones en todos los puntos.

No plantear el equilibrio de tensiones en todas las zonas de la sección.

No estudiar todas las zonas de la pieza.

Como botón de muestra indicamos a continuación, comparativamente con los resultados de nuestra teoría, algunos de los defectos y contradiciones de los criterios clásicos.

\section{Criterio clăsico}

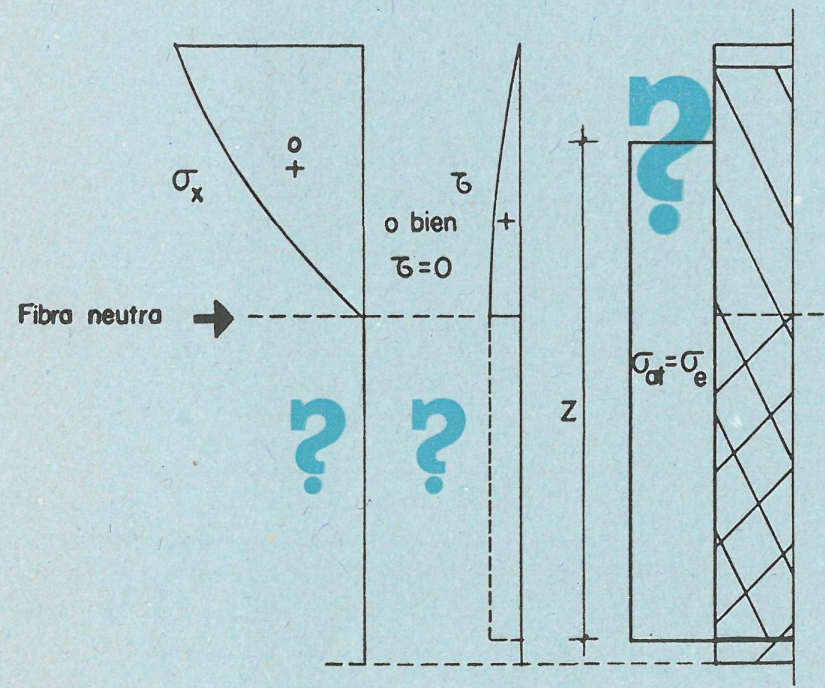

Deformaciones: PARTE.

Solamente se consideran las normales horizontales.

Compatibilidad: NO SE CUMPLE.

No es posible que las deformaciones de los estribos sobrepasen por encima de la fibra neutra las deformaciones admisibles del hormigón no fisurado. nueva feoria

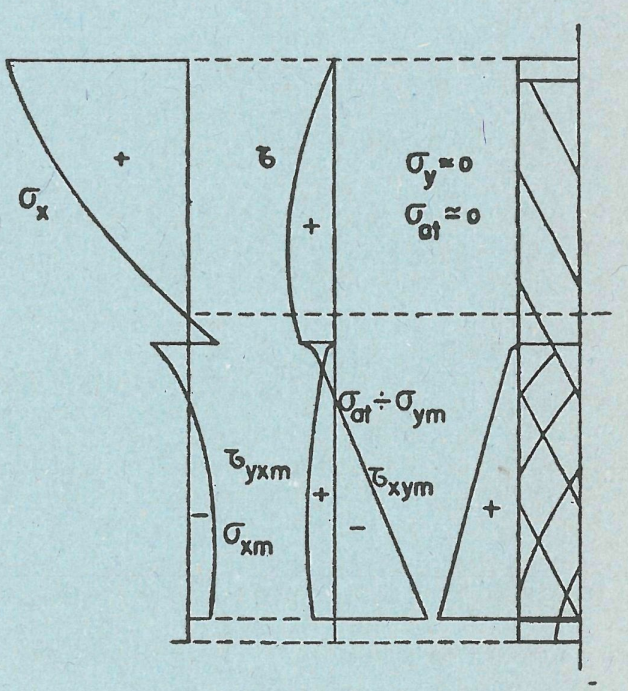

Deformaciones: TODAS.

Normales horizontales y verticales, y tangenciales.

Compatibilidad: SI SE CUIMIPLE.

Las tensiones de los estribos quedan limitadas a partir de la fibra neutra por las deformaciones admisibles del hormigón no fisurado. 
Equilibrio de tensiones: INCOMCPLETO.

En la zona no fisurada, se ignoran las tensiones tangenciales del hormigón, al sobrepasarse la tensión admisible.

En las zonas fisuradas, no se consideran las tensicnes eficaces del hormigón fisurado.

Zonas de la pieza: SOLO UNA.

Se estudia sólo la zona de flexión dominante, ig. norando las zonas próximas a los extremos y a los puntos de inflexión.

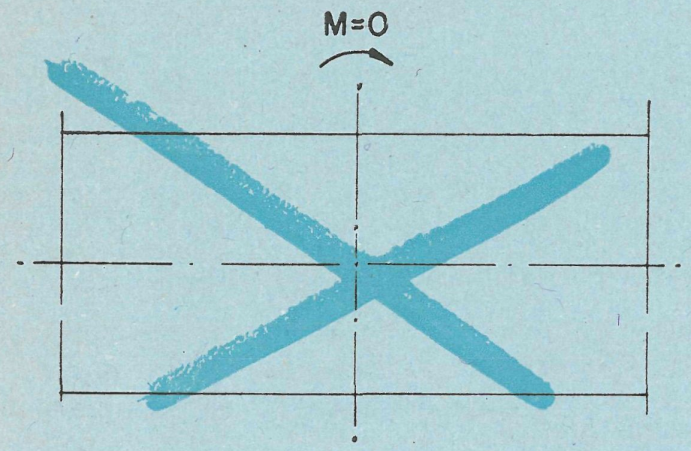

Equilibrio de tensiones: COMPLETO.

En la zona no fisurada, se estudia el equilibrio de todas las tensiones internas del hormigón.

En las zonas fisuradas se estudia el equilibrio en tre las tensiones de los estribos y las eficaces del hormigón fisurado.

Zonas de la pieza: TODAS.

Se estudian tanto la zona de flexión dominante como las de esfuerzo cortante dominante, próximas a los extremos y a los puntos de inflexión.

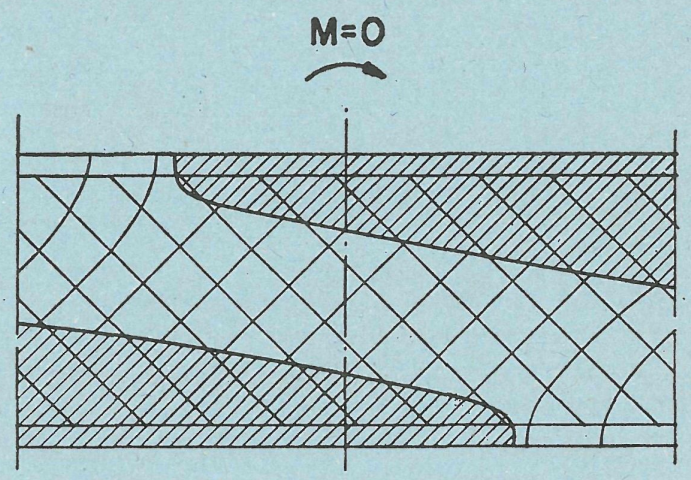

Nosotros creemos que para enfocar correctamente este problema-si bien de forma aproximada, dada la complejidad del asunto-, es indispensable plantearlo mediante la aplicación de las leyes de la Resistencia de Materiales, considerando el equilibrio de tensiones internas y la compatibilidad de las deformaciones, tanto en las zonas en que el hormigón no llega a fisurarse como en las que sufren una intensa fisuración.

En las zonas de hormigón comprimido se deben tener en cuenta, además, las tensiones pulsátiles que transmiten las barras de la celosía constituida por los estribos y las fajas curvas de hormigón comprendidas entre las fisuras, resultando del equilibrio total en aquellas zonas unas determinadas leyes de tensiones normales y tangenciales.

Por otra parte, hay que estudiar el equilibrio de tensiones en las celosias mencionadas, partiendo de una ley razonable de tensiones en los estribos, y del trazado de las fisuras; de esta forma se pueden llegar a obtener unas leyes aproximadas de tensiones eficaces en el hormigón fisurado.

Las tensiones en el hormigón vienen limitadas en rotura, por la aplicación del concepto de resistencia intrínseca. Las del acero de las armaduras longitudinales vienen fijadas por las deformaciones correspnndientes horizontales, así como las de las armaduras transversales (estribos) vienen fijadas principalmente por las horizontales y verticales, viniendo limitadas por las tensiones de agotamiento. De esta forma se plantea el dimensionamiento en rotura resultando del equilibrio de solicitaciones externas y tensiones internas unos sistemas de ecuaciones que, aunque bastante complejos, resuelven teóricamente el problema.

Se estudian los tres estados que pueden presentarse en la pieza: no fisuración, fisuración en zonas de esfiuerzo cortante dominante y fisuración en zonas de flexión dominante. En los estados citados en primero y último lugar se admite la hipótesis de deforinación horizontal plana; en el segundo, correspondiente a secciones próximas a los puntos de inflexión, se admite que la ley de deformaciones en la zona central fisurada sea parabólica.

Quedan, sin embargo, por estudiar las tensiones prácticas. de fisuración según las diferentes fibras de la sección y la disposición y cuantía de las armaduras. El Instituto Eduardo Torroja de la Construcción y del Cemento, con la colaboración de la Empresa Dragados y Construcciones en lo que respecta al necesario cálculo electrónico, ha iniciado un programa de ensayos para contrastar esta teoria y determinar aquellas tensiones y los parámetros de aplicación práctica. La teoría es de aplicación a cualquier forma de sección de la pieza, pero el estudio se ha concretado al caso de sección rectangular. 